April 16, 2021

\title{
Evolutionary dynamics of asexual hypermutators adapting to a novel environment
}

Wei-Chin $\mathrm{Ho}^{1 *}$, Megan G. Behringer ${ }^{1,2,3}$, Samuel F. Miller ${ }^{1}$, Jadon Gonzales ${ }^{1}$, Amber Nguyen ${ }^{1}$, Meriem Allahwerdy ${ }^{1}$, Gwyneth F. Boyer ${ }^{1}$, Michael Lynch ${ }^{1 *}$

${ }^{1}$ Center for Mechanisms of Evolution, The Biodesign Institute, Arizona State University, Tempe, AZ 85287.

${ }^{2}$ Department of Biological Sciences, Vanderbilt University, Nashville, TN 37232.

${ }^{3}$ Department of Pathology, Microbiology, and Immunology, Vanderbilt University Medical Center, Nashville, TN 37232.

*Authors for Correspondence:

Wei-Chin Ho

Center for Mechanisms of Evolution, The Biodesign Institute, Arizona State University, Tempe, AZ 85287.

Phone: 480-727-8005

Email:weichinh@asu.edu

Michael Lynch

Center for Mechanisms of Evolution, The Biodesign Institute, Arizona State University, Tempe, AZ 85287. Phone: 480-965-0868

Email: mlynch11@asu.edu 


\section{Abstract}

2 How microbes adapt to a novel environment is a central question in evolutionary biology. While

3 adaptive evolution must be fueled by beneficial mutations, whether higher mutation rates facilitate

4 the rate of adaptive evolution remains unclear. To address this question, we cultured Escherichia

5 coli hypermutating populations, in which a defective methyl-directed mismatch repair pathway

6 causes a 140-fold increase in single-nucleotide mutation rates. In parallel with wild-type E. coli,

7 populations were cultured in tubes containing Luria-Bertani broth, a complex medium known to

8 promote the evolution of subpopulation structure. After 900 days of evolution, in three transfer

9 schemes with different population-size bottlenecks, hypermutators always exhibited similar levels

10 of improved fitness as controls. Fluctuation tests revealed that the mutation rates of hypermutator

11 lines converged evolutionarily on those of wild-type populations, which may have contributed to

12 the absence of fitness differences. Further genome-sequence analysis revealed that, although

13 hypermutator populations have higher rates of genomic evolution, this largely reflects the effects

14 of genetic draft under strong linkage. Despite these linkage effects, the evolved populations exhibit

15 parallelism in fixed mutations, including those potentially related to biofilm formation,

16 transcription regulation, and mutation-rate evolution. Together, these results generally negate the

17 presumed relationship between high mutation rates and high adaptive speed of evolution,

18 providing insight into how clonal adaptation occurs in novel environments.

19 Key words: adaptation; bottleneck effects; drift barrier; Escherichia coli; mutational load;

20 mutation rate. 


\section{Significance statement}

22 While mutations are critical source for the adaptation in a new environment, whether or not the

23 elevated mutation rates can empirically lead to the elevated adaptation rates remains unclear,

24 especially when the environment is more heterogenous. To answer this question, we evolved $E$.

25 coli populations with different starting mutation rates in a complex medium for 900 days and

26 then examined their fitness and genome profiles. In the populations that have a higher starting

27 mutation rate, despite faster genome evolution, their fitness improvement is not significantly

28 faster. Our results reveal that the effect of elevated mutation rates is only very limited, and the

29 mutations accumulated in hypermutators are largely due to linkage effect. 


\section{Introduction}

Beneficial mutations are the ultimate source of adaptive evolution. Therefore, it is of interest to study how changes to mutational processes can influence an adaptive process. In terms

33 of mutation rates, a theoretically complicated relationship with the rate of adaptation in asexual

34 populations was proposed in the early studies on the evolution of sex (Muller 1932; Crow and

35 Kimura 1965). These studies posit that, when mutation rates are low, such that the waiting time

36 for a beneficial mutation to arise in a population remains long, increases in the mutation rate can

37 result in linear increases in the adaptation rate. In contrast, when mutation rates are relatively high, such that multiple beneficial mutations frequently arise in different individuals within a population, beneficial mutations may interfere with each other's opportunity to spread through the entire population. Thus, the facilitation effect of the mutation rate on the adaptation rate becomes

41 diminished, a phenomenon later called clonal interference (Gerrish and Lenski 1998). More recent

42 theoretical studies have suggested that the effective number of beneficial mutations per population

43 is critical for the strength of clonal interference, and effective population sizes and effect-size

44 distributions of mutations have also been proposed to be influential (Gerrish and Lenski 1998;

45 Wilke 2004; Kim and Orr 2005; Bollback and Huelsenbeck 2007; Desai and Fisher 2007; Park

46 and Krug 2007; Campos and Wahl 2010; Park et al. 2010; Good et al. 2012; Penisson et al. 2017).

47 The relationship between mutation rates and rates of adaptation is further complicated

48 because mutation rates can be plastically different in various environments (Williams and Foster

49 2012; Long et al. 2016; Shewaramani et al. 2017) and evolve over time (Wielgoss et al. 2013;

50 Swings et al. 2017). Given that most mutations are deleterious, high mutation rates create high

51 mutational loads in the genome, potentially driving the spread of antimutator alleles and resulting

52 in the evolution of lower mutation rates (Muller 1950; Kimura 1967; Lynch 2008). According to 
53 the drift-barrier hypothesis, the reduction of the mutation rate should continue until the addition of

54 new antimutator alleles no longer contributes to a significant enough reduction in mutational load

55 to overcome genetic drift (Lynch 2010b; Sung et al. 2012; Lynch et al. 2016). Consequently, if

56 two populations with initially different mutation rates adapt to the same constant environment, the

57 resulting difference in fitness-improvement rates can be less than predicted if both populations

58 converge evolutionarily to similar mutation rates.

While ample theoretical discussion exists on the relationship between mutation rates and

60 the rate of adaptation, the theory has been mostly focused on constant environments with a simple

61 fitness landscape, neglecting the likely complexity of more natural environments. The limited

62 empirical evidence in asexual populations suggests that adaptation rates are a concave-down

63 function of the mutation rate (Arjan et al. 1999; Desai et al. 2007; Sprouffske et al. 2018). However,

64 the experimental environments in these studies were generally simple and homogeneous (Arjan et

65 al. 1999; Desai et al. 2007; Sprouffske et al. 2018), or the populations of interest were already

66 well-adapted to the experimental environment (McDonald et al. 2012). How the relationship

67 between mutation rates and adaptation rates evolves when the environmental setting becomes more

68 complex and heterogeneous is less understood. For example, the modes of adaptation in complex

69 environments may vary greatly due to the presence of a more rugged fitness landscape, additional

70 paths available for fitness improvement, the presence of multiple spatial or nutritional niches, or

71 more complicated genetic interactions. (Handel and Rozen 2009; Lynch 2010a; Ochs and Desai

72 2015; Guo et al. 2019). Therefore, it is necessary to study adaptive processes in more complex and

73 heterogeneous environments to determine whether the principles observed in simpler

74 environments still apply. 
To study how the mutation rate affects adaptation in a more complex setting, we performed long-term experimental evolution of Escherichia coli in culture tubes containing a complex medium, Luria-Bertani (LB) broth, which is comprised of a nutritionally-rich mixture of multiple amino-acid based carbon sources (Sezonov et al. 2007). In contrast to evolution in flasks containing glucose-limited media, such environments can facilitate the rapid emergence of stable subpopulations and clonal divergence based on spatial niche differentiation and amino-acid populations $(\mathrm{MMR}+)$ and hypermutator populations with an impaired methyl-directed mismatch repair pathway (MMR-, obtained by mutL knockout), for which the single-nucleotide mutation rate is 140 -fold higher than that for the WT genetic background (Lee et al. 2012). Because the results of experimental evolution may be altered by different demographic settings (Vogwill et al. 2016; Wein and Dagan 2019), replicated populations were assigned to one of three different daily-

87 transfer size treatments: 1/10 (large, L), 1/10 (medium, M), and 1/10 (small, S) dilutions into fresh media. Here, we examined the differences in phenotypic and molecular evolution among these populations over the course of 900 days.

\section{$91 \quad$ Results}

92 Higher initial mutation rates do not translate into faster rates of fitness improvement. When

93 batch cultured, E. coli commonly adapt to their experimental environments and show fitness

94 improvement compared to their ancestors (Van den Bergh et al. 2018; McDonald 2019). To

95 compare adaptation rates in populations originating from genetic backgrounds with different initial

96 mutation rates (MMR- and WT), we performed head-to-head competition assays between

97 populations that had evolved for 900 days and their corresponding ancestor. For each of six 
genetic-background/transfer-size combinations $(2 \times 3)$, four replicated populations were measured. Across all 21 populations with data available (three were aborted; see Materials and Methods), mean fitness significantly increased relative to the time-zero ancestor, by a ratio of 1.14 ( $\mathrm{SE}=$

$1010.019 ; P=5.8 \times 10^{-7}$, two-tailed $t$-test), indicating the evolution of these populations shaped by 102 adaptive processes.

104 from that for WT populations among any of the transfer sizes (Fig. 1A; L: $P=0.26$; M: $P=0.46$;

$105 \mathrm{~S}: P=0.15$, nested ANOVA). In particular, considering the ratio of mean fitness improvement 106 (MMR-: WT), no transfer size produced a ratio significantly different from 1.0. For example, for 107 the L transfer size, the mean fitness improvement for MMR- and WT backgrounds are respectively $1080.27(\mathrm{SE}=0.043)$ and $0.21(\mathrm{SE}=0.047)$, and therefore the ratio $(\mathrm{MMR}-\mathrm{WT})$ is $1.28(\mathrm{SE}=0.35)$.

109 Similarly, in the $\mathrm{M}$ and $\mathrm{S}$ transfer sizes, the ratios are $0.69(\mathrm{SE}=0.28)$ and $2.76(\mathrm{SE}=1.98)$, 110 respectively. Thus, starting evolution as a hypermutator does not necessarily translate into a faster 111 fitness-improvement rate.

Previous studies of E. coli in simpler, more homogeneous environments have shown that 113 the most rapid increases in population fitness typically occur within 2500 generations, after which 114 the rate of adaptation significantly slows (Barrick et al. 2009). At 900 days, the L populations, 115 which due to their large transfer size experienced the least number of cell divisions, had 116 experienced $\sim 3000$ generations, whereas the $S$ populations had experienced $\sim 21,000$ generations.

117 As such, the absence of significant differences in the cumulative amount of adaptation over this 118 period - despite large differences in initial mutation rate - might be a consequence of both genetic 119 backgrounds having exited an initial period of rapid fitness evolution. Thus, to better survey any 120 temporal heterogeneity in the rate of adaptation, we further assessed fitness after 90-, 300-, and 
121 600-days of evolution in response to $\mathrm{L}$ and $\mathrm{S}$ transfer sizes. The results of nested ANOVA again

122 demonstrate a lack of evidence for an increase in initial mutation rates leading to an increase in

123 the amount of fitness improvement (Fig. 1B-D). The results are not qualitatively changed even

124 when the natural-logarithmic transformed fitness is used in the analysis (Fig. S1). Thus, high

125 mutation rates did not result in accelerated fitness improvement in these asexual populations even

126 in the early stages of adaptation.

128 Mutation rates evolve to be more similar throughout experimental evolution. The indifference

129 of adaptation rates to initial mutation rates might be explained if the mutation rates of hypermutator

130 and WT populations became more similar during the evolution experiment, either due to a

131 reduction in the mutation rate in initially hypermutating populations or to an increase of the rate

132 in WT populations. To test this possibility, we performed fluctuation tests, which indirectly 133 measure mutation rates at a resistance locus (Foster 2006), on different clones isolated from

134 evolved populations after 900 days. Although the ratio of rifampicin-resistance mutation rates for

135 the two ancestral lines (MMR-: WT) was 250 (Fig. S2), after 900 days of evolution, the mean

136 difference in mutation rates between MMR- and WT backgrounds greatly decreased across all

137 transfer sizes (Fig. 2). For example, in the L transfer size, the mean mutation rate is $5.0 \times 10^{-7}(\mathrm{SE}$

$\left.138=2.7 \times 10^{-7}\right)$ and $1.6 \times 10^{-8}\left(\mathrm{SE}=1.2 \times 10^{-8}\right)$ for MMR- and WT evolved populations, respectively.

139 Therefore, the ratio of mean mutation rates (MMR- : WT) was reduced to $32(\mathrm{SE}=29)$. This

140 reduction, however, seems mostly attributed to the occasional emergence of higher mutation rates

141 in the WT background as only one population (115) among four tested WT, L populations shows

142 a significant increase of the mutation rate in both tested clones. In the $\mathrm{M}$ and $\mathrm{S}$ transfer size, the

143 ratio of mean mutation rates (MMR- : WT) was also reduced to $32(\mathrm{SE}=15)$ and $12(\mathrm{SE}=3.9)$, 
144 respectively. However, the repeated evolution of lower mutation rates in clones isolated from

145 MMR- background plays a more important role in the reduction as all of the tested clones from

146 MMR-, M or S populations show a significant decrease of the mutation rate. Thus, the evolutionary

147 convergence of mutation rates likely contributes to the difference in adaptation rates being less

148 than what might be expected based on initial differences in mutation rates, but interestingly, the

149 specific evolutionary mechanisms underlying these similarities are different. These observations

150 demonstrate how transfer schemes can affect the evolutionary dynamics of mutation rates in

151 asexual populations.

Genome evolution rates are less different than predicted by initial mutation rates. To enhance

154 our understanding of how the tempo and mode of genomic evolution relate to fitness and

155 phenotypic evolution, we performed metapopulation sequencing of each experimental population

156 roughly every 100 days to acquire mutation profiles of associated derived allele frequencies

157 (DAFs). For each combination of genetic background and transfer size, we estimated the rate of

158 genomic evolution by regressing the number of mutations per clone (i.e., the sum of DAFs of all

159 observed SNPs) of all populations against the number of generations at each sampled time point.

160 Because a quadratic regression model did not significantly outperform the linear regression $(P=$

1610.028 for MMR-/L; $P>0.10$ for the others, nested ANOVA), we will focus on the results of the

162 linear regression below.

163 As with the rate of adaptation, for all three transfer sizes, the ratio of the rate of genomic

164 evolution between the two backgrounds (MMR-: WT) was much smaller than the initial difference

165 in mutation rates (Fig. 3A). For example, under the L transfer size, the rate of genomic evolution

166 is $115(\mathrm{SE}=5.3)$ and $30(\mathrm{SE}=4.3)$ mutations per clone per 1000 generations for MMR- and WT 
167 populations, respectively. Therefore, the ratio of the genomic-evolution rates is only 3.8 ( $\mathrm{SE}=$

168 0.58). Similarly, in the $\mathrm{M}$ and $\mathrm{S}$ transfer sizes, the ratio is $20(\mathrm{SE}=1.3)$ and $23(\mathrm{SE}=1.6)$,

169 respectively. This observation remained qualitatively similar even when different kinds of

170 measurements for genomic divergence were used, e.g., the number of detected mutations (Fig.

171 S3A), nucleotide diversity (Fig. S3B), or when genomic divergence was estimated only by 172 synonymous SNPs (Fig. S4).

To further survey how genomic evolution rates vary across experimental populations and

174 to determine if the mean rates accurately represent the majority of experimental populations, we

175 separately measured the rate of genomic evolution for each experimental population (Fig. S5).

176 This revealed that the distribution of rates in the WT populations under the L transfer size is wider

177 than the distributions of rates in all other combinations of genetic background and transfer sizes.

178 Specifically, in the L transfer size, although five of the eight WT populations exhibit a genomic

179 evolution rate of $\sim 10$ mutations per clone per 1000 generations, one WT population (115) has a

180 rate about $2 \times$ higher, and two WT populations (101 and 113 ) have a rate close to 100 , similar to

181 MMR- populations. Consistent with the results of fluctuation tests noted above, these results

182 suggest that some, but not all, WT populations under the L transfer size evolved a higher mutation

183 rate (see Discussion).

185 Mutations arising in hypermutators are more likely to be fixed. Using longitudinal

186 metagenomics-sequencing data allows one to observe evolutionary dynamics at the level of single

187 mutations and thus better understand the entire adaptive process. Here, we will focus on fixed

188 mutations because they are more likely to contribute to adaptive processes than polymorphic

189 mutations or other mutations that are transient in a population. Because our experimental- 
190 environment setting facilitates the development of subpopulation structure, we applied clade-

191 aware hidden Markov chain (caHMM) analysis. Assuming coexistence of two clades (major and

192 minor) in the population, caHMM considers each mutation's DAFs found in different sequencing

193 time-points and then infers which clade each mutation belongs to, whether each mutation reaches

194 within-clade fixation, and when a fixed mutation reaches fixation (Good et al. 2017).

195 One characteristic parameter in an adaptive process is the probability that mutations reach

196 fixation in a population. With the observed numbers of both detected and fixed mutations, we first

197 tested whether hypermutator populations have a different probability of mutation fixation within 198 clades compared to WT populations. Different mutation types have a different potential to impact 199 populational fitness. For example, compared to synonymous SNPs, nonsynonymous SNPs have a 200 greater potential to change protein functions, intergenic SNPs have a greater potential to change 201 protein expression, and structural variations (SVs; including indels and mobile-element insertions) 202 have a greater potential to disrupt a protein. Therefore, we performed separate tests on the 203 conditional fixation probability for these four functional categories of mutations.

While not always significant, the fixation probability in the MMR-populations is generally higher than in WT populations across different transfer sizes and different categories of mutations (Fig. 4A). The fixation probability is similar across different categories of mutations, regardless 207 of their perceived potential to affect fitness, suggesting that the fixation of most mutations is a 208 consequence of genetic hitchhiking as opposed to intrinsic beneficial effects.

Another critical factor determining the temporal dynamics of a mutation is the underlying 210 fitness effect. With the temporal data of allele frequencies for a mutation, we can quantify the net 211 selection coefficient, which reflects its own fitness effects but can be potentially affected by the 212 effects of linked mutations. We did not find a significant difference between the selection 
213 coefficients of fixed mutations in the two genetic backgrounds (Fig. 4B). More importantly, we

214 also found that different categories of mutations show similar mean selection coefficient estimates,

215 which again suggests a pivotal contribution of hitchhiking effects to the mutational dynamics of

216 genome evolution these in asexual populations.

218 Neutrality tests reveal partial evidence for positive selection. In theory, comparing the number

219 of fixed mutations in functional categories of sites with different potential effects on fitness can

220 summarize general patterns in the mode of genome evolution (McDonald and Kreitman 1991;

221 Rand and Kann 1996). If a population has experienced strong positive selection on protein function

222 or expression, it is expected that there will be more fixed mutations with a greater potential to

223 change protein function or expression than mutations with smaller such potential. If a population

224 has experienced purifying selection on protein function or expression, it is expected that there will

225 be less fixed mutations with a greater potential to change protein function or expression than

226 mutations with smaller such potential. Therefore, the ratio of the number of fixed nonsynonymous

227 synonymous SNPs $\left(F_{\mathrm{N}}\right)$ to the number of fixed synonymous SNPs $\left(F_{\mathrm{S}}\right)$ or the ratio of the number

228 of fixed intergenic SNPs $\left(F_{\mathrm{I}}\right)$ to $F_{\mathrm{S}}$ is predicted to be large with a strong positive selection.

229 Similarly, $F_{\mathrm{N}} / F_{\mathrm{S}}$ or $F_{\mathrm{I}} / F_{\mathrm{S}}$ are predicted to be small with a strong purifying selection. However,

230 these ratios are not directly comparable in MMR- and WT backgrounds, because the two genetic

231 backgrounds have varied mutational spectra and different relative rates of nonsynonymous and

232 synonymous mutations (Lee et al. 2012). To address this issue, we normalized the observed $F_{\mathrm{N}} / F_{\mathrm{S}}$

233 by the ratio of nonsynonymous and synonymous mutations $\left(U_{\mathrm{N}} / U_{\mathrm{S}}\right)$ previously observed in a

234 mutation accumulation experiment which utilized our exact ancestral genotypes (Lee et al. 2012).

235 The ratio of these ratios, $\left(F_{\mathrm{N}} / F_{\mathrm{S}}\right) /\left(U_{\mathrm{N}} / U_{\mathrm{S}}\right)$, will be referred to as the neutrality index of 
nonsynonymous SNPs, since being significantly $>1.0$ or $<1.0$ implies a predominance of positive selection in driving mutation fixation or a predominance of purifying selection in driving mutation extinction. It is analogous to Tachida's index for neutrality (Tachida 2000) but with a different normalizing approach - via results from mutation-accumulation experiment instead of polymorphism. This definition of the neutrality index can be applied to any kind of mutations. For example, we also define the neutrality index of intergenic SNPs as $\left(F_{\mathrm{I}} / F_{\mathrm{S}}\right) /\left(U_{\mathrm{I}} / U_{\mathrm{S}}\right)$, where $U_{\mathrm{I}}$ is the number of intergenic mutations observed in mutation accumulation (Lee et al. 2012). size is significantly larger than one, consistent with the model of strong positive selection (Fig. 4C). Moreover, the neutrality index of intergenic SNPs in MMR-populations under all transfer

246 sizes is significantly smaller than one, suggesting overall strong purifying selection on intergenic

247 SNPs (Fig. 4D). On the contrary, the neutrality index in WT populations is never significantly 248 different from one. While this can indicate a weaker strength of selection in WT populations, it 249 may also be the result of insufficient statistical power due to an overall smaller number of fixed

250 SNPs in WT populations, evident by their large confidence intervals associated with the point 251 estimation.

253 Parallel evolution of fixed mutations at the genic and nucleotide level. As the previous analysis

254 suggests that the statistical power of the neutrality index to reveal positive selection may be 255 insufficient, we also considered parallelism of fixed mutations to examine the action of positive 256 selection, using two different metrics. First, we utilized a sum of $G$-scores to measure the excess 257 parallelism across fixed nonsynonymous mutations relative to the expectation based on gene 258 lengths (Tenaillon et al. 2016). For each gene, a higher $G$-score implies that the gene is more 
enriched for fixed nonsynonymous mutations than expected by gene length. We then summed the

$260 G$-scores of all genes for each combination of genetic background and transfer size and found

261 statistical significance in all cases ( $z$-test, Fig. 5A). Second, we calculated the mean Bray-Curtis

262 similarity of the number of fixed nonsynonymous mutations across all genes (Turner et al. 2018)

263 for all pairwise comparisons of evolved populations in each combination of genetic background

264 and transfer size. The results again show statistical significance for similarity in all cases $(z$-test,

265 Fig. S6). Therefore, both findings suggest that positive selection has shaped the genomic evolution

266 of populations in all genetic-background / transfer-size combinations.

267 The analysis of parallelism also helps reveal which mutations are most likely to be drivers

268 of adaptation. Using the same $G$-score analysis, in each genetic-background/transfer-size

269 combination, we identified genes that were overrepresented for fixed nonsynonymous mutations

$270(P<0.05$, Bonferroni correction; Fig. 5B). Gene Ontology (GO) analysis on these gene subsets

271 revealed that significantly enriched GO terms were often related to transcription regulation and

272 biofilm formation (Table S1). Using similar methods, we also identified subsets of genes enriched

273 for fixed mutations in intergenic regions (Fig. 5C) and for structural mutations, including indels

274 and IS-element insertions (Fig. 5D). Together, the genes in these lists serve as good candidates for

275 revealing the various mechanisms important to adaptation in complex environments (see

276 Discussion).

277 In addition to genic-level parallelism, we also identified nucleotide-level parallelism. In

278 particular, 197 cases of parallel fixed nonsynonymous mutations were identified in at least two

279 experimental populations within the same combination of transfer size and genetic background

280 (Table S2). Because the probability that fixed mutations would occur by chance at a given

281 nonsynonymous site in at least two experimental populations is very low, the observed parallelism 
282 at the nucleotide-level again suggests positive selection and identifies important candidates for

283 studying the molecular mechanisms associated with adaptation in complex media. For example,

284 three instances of parallel mutation within fim $H$ were located in its mannose-binding domain, and

285 therefore may be good candidates for future functional studies of cell-adhesion (Schembri et al.

286 2001). Furthermore, three instances of parallel-fixed nonsynonymous mutations were found in

287 genes with GO terms associated DNA repair or DNA replication, including dnaE (DNA pol III

288 subunit $\quad \alpha$ ), yajL (protein/nucleic acid deglycase 3), and $n r d A$ (ribonucleoside-diphosphate

289 reductase $1, \alpha$ subunit dimer). As all three of these cases arose in $\mathrm{M}$ and $\mathrm{S}$ transfer sizes with

290 MMR- backgrounds, they may be good candidates for studying the molecular mechanisms

291 associated with lowering mutation rates.

\section{Discussion}

294 Here, we describe the effect of the initial mutation rate on the rate of fitness improvement and 295 genomic evolution by experimentally evolving E. coli with two distinct mutation-rate backgrounds.

296 While the initial difference in mutation rates of these two genetic backgrounds is $>100 \times$, after 900

297 days of evolution, the differences in the net rates of fitness improvement (0.7-2.7 fold), in the

298 mutation rates (12-32 fold), and in level of genome evolution (4-23 fold) are much smaller. At the

299 resolution of single mutations, we found that mutations arising in MMR-populations exhibit

300 higher fixation probabilities than mutations in WT populations, while mutations in different

301 categories with presumably different fitness effects show similar fixation probabilities within each

302 genetic-background treatment, suggesting the strong influence of hitchhiking effects in genome

303 evolution in these populations. Despite the strong linkage and pervasive genetic draft, there is still

304 evidence, in the form of a high degree of parallelism in mutations arising in particular genes and 
305 nucleotides, that positive selection shapes genome evolution in all transfer-size/genetic-

306 background combinations. The observed mutations with high parallelism also serve as excellent

307 candidates for understanding the mechanisms of adaption to the complex conditions provided by

308 the experimental environment.

310 Effects of high mutation rates on evolution. Our experiments reveal that, even for 900 days of

311 evolution in a complex medium, hypermutating E. coli does not necessarily exhibit a faster rate of

312 adaptation than wild-type E. coli. This observation is consistent with previous experimental-

313 evolution results over a shorter period and in simpler media, such as glucose medium DM25 for

3141000 generations (Arjan et al. 1999) and glucose medium DM1000 for 3000 generations

315 (Sprouffske et al. 2018). Our experiments further demonstrate that populations with initially

316 hypermutator backgrounds can rapidly evolve lower mutation rates. Together with other empirical

317 work on prokaryotic (Sprouffske et al. 2018) and eukaryotic hypermutators (McDonald et al. 2012),

318 these results suggest that the strong genetic load due to deleterious mutations remains a pivotal

319 factor in the evolution of mutation rates, consistent with the drift-barrier hypothesis (Lynch 2010b;

320 Sung et al. 2012). Even when some new hypermutator alleles can spread in a population by linkage

321 with other beneficial mutations during the adaptive process and thus briefly improve the rate of

322 adaptation (Sniegowski et al. 1997; Tenaillon et al. 1999; Tenaillon et al. 2001), such events are

323 usually transitory (Giraud et al. 2001; Desai and Fisher 2007; Wielgoss et al. 2013). Moreover, the

324 fact that lowering the mutation rate could not have involved a reversion of deleted MMR in our

325 experiments implies that there is excess capacity for improving replication fidelity through other

326 parts of E. coli genome. As mutation-rate evolution occurred over a relatively short period in our 
study, the results bear on several critical questions for future studies, including the rapidity of the dynamics of the evolution of mutation rates and the consequences for the mutational spectrum.

Effect of genetic linkage in evolution. In asexual populations with reduced recombination, the

331 fate of a mutation is largely affected by its association with other mutations due to strong genetic

332 linkage (Gillespie 2000; Neher 2013; Couce et al. 2017). Extensive hitch-hiking is a feature of our

333 evolving E. coli populations, as we observe similar fixation probabilities and net associated fitness

334 effects for fixed mutations across different functional categories of mutations. To better illustrate

335 this effect of genetic linkage, we showed that the temporal DAF changes of any two SNPs in the

336 same genome are highly correlated (i.e., a rightly-skewed distribution of correlation coefficients)

337 and result in more largely positive correlation coefficients than for a random expectation of non-

338 linked mutations (Fig. S7). Accordingly, pre-existing non-beneficial mutations can become fixed

339 by hitchhiking with newly-arising beneficial mutations, and these linkage effects can limit the rate

340 of adaptation (Schiffels et al. 2011; Kosheleva and Desai 2013). Therefore, even though MMR-

341 populations show a range of genome-evolution rates $4-23 \times$ higher than WT populations, the

342 excess of fixed mutations does not directly contribute to adaptation rates.

344 Effects of transfer sizes in evolution. The three different transfer sizes (L, M, and S) implemented

345 in our evolution experiment allow us to compare adaptive processes in different population-genetic

346 environments. In theory, when the transfer size is large, reduced genetic drift renders a higher

347 efficiency of promotion of beneficial mutations in a population. Consistent with this theoretical

348 prediction, the populations cultured with the L-transfer size show highest rates of fitness gain (Fig.

349 1), for nonsynonymous mutations, the observed neutrality index of MMR- populations under L 
350 transfer size is lowest and $<1.0$ (Fig. 4C). Compared to L-transfer treatment, the populations

351 cultured with the S-transfer size may have accumulated more deleterious mutations and thus show

352 less fitness improvement.

353 We also found that the populations under the L-transfer size tend to evolve higher mutation

354 rates (Fig. 2), as hypermutator alleles may hitchhike with more selective sweeps of beneficial

355 mutations. Previous research has also demonstrated that the spread of hypermutator alleles tends

356 to be found in populations with a larger population size or weaker bottleneck effects (Raynes et al.

357 2014). As a result, even though the mutation rates of lines in two genetic backgrounds (WT and

358 MMR-) evolved to be closer during the experiments with all three transfer sizes, the driving

359 mechanisms are different between populations under the $\mathrm{L}$ transfer size and those under $\mathrm{M}$ or $\mathrm{S}$

360 transfer sizes (Fig. 2).

361 In theory, the evolved populations under larger transfer sizes experienced a relatively

362 longer duration of stationary phase between transfers as the number of divisions needed to reach

363 the stationary phase are smaller. Given that the physiological features of E. coli can be different in

364 different growth phases (Pletnev et al. 2015), the evolutionary pressure under different transfer-

365 sizes may also be different. Therefore, interpreting any results based on the comparison across

366 transfer-size treatments needs more caution. In our case, however, such differences are likely

367 limited, as we still found significant parallelism in the fixed enriched mutations across different

368 transfer sizes (Fig. S8). Whether there is any adaptation specific to the different growth phases or 369 different combinations of growth phases is a subject for future studies.

371 The possible role of biofilm formation in adaptation. With respect to the specific genes and 372 biological processes that appear to be targets for adaptation, our analysis of candidate genes 
373 suggests that biofilm formation is an important characteristic in adapting to the complex setting

374 imposed by the experimental environment. In particular, the formation of type I fimbriae is critical

375 for biofilm formation in E. coli (Pratt and Kolter 1998). Consistently, several genes enriched for

376 fixed nonsynonymous mutations are related to formation of type I fimbriae (Fig. 5B), including

$377 \mathrm{fimH}$ and $\mathrm{fim} G$, which account for components the type I fimbriae (Waksman and Hultgren 2009;

378 Le Trong et al. 2010), fimB and fimE, which regulate the expression of fimAICDFGH operon

379 (Olsen et al. 1998), and proQ (RNA chaperone) and lsrK (autoinducer-2 kinase), which also

380 facilitate biofilm formation (Li et al. 2007; Sheidy and Zielke 2013). We additionally observed an

381 enrichment of mutations in the intergenic region of fimE/fimA (Fig. 5C) which contains a phase-

382 variable promoter for regulating the expression of the fimAICDFGH operon (Abraham et al. 1985;

383 Spears et al. 1986). Lastly, the list of genes enriched with structural mutations (Fig. 5D) also

384 include fimE, which primarily turns off the expression of fimAICDFGH operon, and several genes

385 related to gatYZABCD operon, including gatZ, gatB, and gatA, whose deletions can increase

386 biofilm formation (Domka et al. 2007). Many of these candidate genes related to type I fimbriae

387 contributed to the adaptation in an earlier experimental environment of a similar nature (Behringer

388 et al. 2018). Moreover, structural mutations in gatZ and gatA genes have been found to contribute

389 to the initial adaptation of $E$. coli in a mouse gut, another example of a complex environment

390 (Barroso-Batista et al. 2014). Studying the genetic variants promoting the evolution of biofilm in

391 complex environments may be of particular interests in the field of public health, as the evolution

392 of biofilm has been considered to be related to the evolution of microbial social behaviors (Tarnita

393 2017), the evolution of pathogenicity (Kaper et al. 2004; Naves et al. 2008; Rossi et al. 2018), and

394 the evolution of antibiotic resistance (Avalos Vizcarra et al. 2016; Sharma et al. 2016). Thus, 

benefits to our experimentally evolved populations.

understanding the evolution of biofilm formation may be key to increasing the efficiency of treatments in patients to combat the fast emergence of antibiotic resistance and pathogenicity.

\section{Candidate transcriptional regulators involved in adaptation. Because we have provided fresh} media every day during the experimental evolution, a fast switch from stationary phase to exponential growth phase may bring benefits to the evolving populations (Monod 1949; Navarro Llorens et al. 2010). Interestingly, several genes enriched in fixed nonsynonymous mutations in our study are transcription regulators, including $\operatorname{arc} A, \operatorname{cad} C, \operatorname{cyt} R, \operatorname{rbs} R, r \operatorname{seB}$, and $\operatorname{ssp} A$ (Fig. 5B). The genes enriched in structured variations also include transcription regulators, such as $\operatorname{arc} B$, $c a d C$, rpoS, and $n l p D$ (Fig. 5C). The mutations on these genes may contribute to the transcriptomic reprogramming for the fast switch from stationary phase to exponential phase. For example, $r s e B$ is a negative regulator of the stationary phase effector, sigma factor E (Missiakas et al. 1997); and both $c y t R$ and $r b s R$ are repressors to the carbon limitation effector, cAMP-CRP (Bell et al. 1986; Mauzy and Hermodson 1992; Kristensen et al. 1996). Therefore, gain-of-function mutations on these three genes can theoretically reduce the chance that cells stay in stationary phase. In addition, $\operatorname{arc} A, \operatorname{arcB}, \operatorname{cad} C, \operatorname{rpoS}$, and $\operatorname{sp} A$ are known as stress-responding activators (Iuchi et al. 1989; Lange and Hengge-Aronis 1991; Watson et al. 1992; Williams et al. 1994; Rolfe et al. 2011). Therefore, loss-of-function mutations on these genes are presumably beneficial in the experimental environment involving a frequent supply of fresh media and presumably imposing low stress. 
417 Concluding Remarks. To sum up, our results reveal that high mutation rates in E. coli have only

418 a very limited influence on the rate of adaptation. Our findings may provide useful insights for

419 clinically relevant processes involving asexual populations, such as the evolution of improved

420 growth rates in pathogens and the emergence of antibiotic resistance in natural or host environment.

421 In particular, our experimental evolution results in complex media are likely to be more

422 representative, as natural or host environments are usually highly heterogeneous. For example, a

423 combination of the occasional emergence of hypermutators under weaker bottlenecks and the

424 consistent evolution of antimutators under stronger bottlenecks may explain why a low to

425 intermediate frequency of hypermutators is usually found in pathogen populations (Couce et al.

426 2016; Veschetti et al. 2020). Whether the elevated mutation rates affect adaptation rates and the

427 pattern of genome evolution in these populations under natural or host environments should be

428 subject to future research. For example, hypermutators may be critical for a founding population

429 in a new environment, especially with epistasis in the fitness landscape, but such effect of

430 hypermutation can diminish after a long time (Mehta et al. 2019).

\section{Materials and Methods}

433 Strains. The ancestral strains used in experimental evolution are descendants of PMF2 and

434 PMF5, provided by the Foster Lab (Lee et al. 2012). PMF2 is a prototrophic derivative of E. coli

$435 \mathrm{~K}-12$ str. MG1655, and its genetic background is called by WT in the paper. PMF5 is derived

436 from PMF2 with mutL deletion, providing the MMR- genetic background. For both kinds of

437 strains, a $3513 \mathrm{bp}$ deletion to the $\operatorname{araBAD}$ operon is further introduced by lambda red

438 recombineering as a neutral marker. Plates with TA agar (1\% Arabinose, 1\% Tryptone, $0.5 \%$

$439 \mathrm{NaCl}, 0.1 \%$ Yeast Extract, 0.005\% TTC (Sigma T8877)) is used for examining the deletion of 
$440 \operatorname{araBAD}$ operon. The colonies with deletion (ara-) appear to be pink; otherwise, the colonies

$441(\mathrm{ara}+)$ appear to be purple.

443 Experimental evolution. When we established the experimental populations, the ancestral

444 strains were first cultivated overnight at $37^{\circ} \mathrm{C}$ on LB agar plates, and then their single-isolated

445 progenitor colonies were inoculated in a $16-\times 100-\mathrm{mm}$ glass tube with $10 \mathrm{~mL}$ of LB-Miller

446 broth (BD Difco). The tubes were cultured at $175 \mathrm{rpm}$ shaking at $37^{\circ} \mathrm{C}$. Every day, cultures are

447 thoroughly-vortexed and transferred into a new tube with $10 \mathrm{~mL}$ of fresh LB broth. Three

448 different transfer sizes are used: $1 \mathrm{~mL}($ large), $1 \mathrm{uL}$ (medium), and $1 \mathrm{~nL}($ small), which correspond

449 to different dilution factors: $10^{-1}, 10^{-4}$, and $10^{-7}$. Initially, for each combination of genetic

450 background and transfer sizes, we set up eight replicate tubes in three groups with different

451 transfer sizes. For preventing cross-contamination, four replicates are ara- and four replicates

452 are $\mathrm{ara}^{+}$. During the experimental evolution, experimental populations at day 90, 200, 300, 400,

$453500,600,700,800,900$ were frozen in $-80{ }^{\circ} \mathrm{C}$ freezers for analysis.

455 Competition assay. When evaluating the fitness of an evolved population (ara-), we inoculated

456 the corresponding frozen sample in a tube with $10 \mathrm{~mL}$ of fresh $\mathrm{LB}$ broth at $37^{\circ} \mathrm{C}$ shaking at 175

457 rpm overnight. We also inoculated the corresponding ancestral population in a tube with $10 \mathrm{~mL}$

458 of fresh LB broth at $37^{\circ} \mathrm{C}$ shaking at $175 \mathrm{rpm}$ overnight. We then put $50 \mathrm{uL}$ aliquot from

459 evolved populations and $50 \mathrm{uL}$ aliquot from evolved populations to into a new tube with $10 \mathrm{~mL}$

460 of LB broth at $37^{\circ} \mathrm{C}$ shaking at $175 \mathrm{rpm}$ for $24-\mathrm{hr}$ competition. Immediately after inoculation

461 (day 0) and after 24-hr competition (day 1), we used plate-counting to determine the colony-

462 forming units (CFU) of both ancestral and evolved strains. Specifically, we serially diluted 
$463100 \mathrm{uL}$ aliquot in $900 \mathrm{uL}$ phosphate buffered saline. To distinguish the evolved population from

464 the ancestors, we plated serially diluted aliquots on TA agar because ara-colonies will appear to

465 be pink, while the ancestral $\left(\mathrm{ara}^{+}\right)$will appear to be purple. The TA plates were then incubated

466 at $37^{\circ} \mathrm{C}$ overnight. We then identified the plate with total colonies 30-300 and counted the

467 number of colonies for the evolved population and the ancestors. Based on the colony numbers

468 and the dilution factor during the serial dilution, we then calculated the CFU of ancestors before

469 the competition $\left(A_{0}\right)$, the CFU of ancestors after the competition $\left(A_{1}\right)$, the CFU of the evolved

470 population before the competition $\left(E_{0}\right)$, and the CFU of the evolved population after the

471 competition $\left(E_{1}\right)$. The fitness of the evolved population $(w)$ relative to the ancestor was then

472 calculated by the following formula:

$$
w=\ln \left(E_{1} / E_{0}\right) / \ln \left(A_{1} / A_{0}\right),
$$

474 which is the ratio of two Malthusian parameters (Lenski et al. 1991).

475 At day 0 , we also serially diluted and plated $100 \mathrm{uL}$ aliquot of evolved population as a

476 control. For an evolved population, if both purple and pink colonies in the countable control

477 plates (30-300 colonies per plate), its data will be discarded from the analysis because we are

478 not sure about the source of the pink colonies in the experimental plates.

480 Fluctuation test and mutation rate estimation. We quantified mutation rates of evolved 481 populations (at day 900) and ancestors by fluctuation tests (Foster 2006). Briefly, fluctuation

482 tests measure the rate of resistance to the antimicrobial rifampicin which is conferred by

483 mutations to $r p o B$. For each combination of genetic background and transfer size, the four ara-

484 populations were assayed. For each population, two biological replicates with different starting

485 clones were assayed. For each of the WT or MMR- ancestor, we also run replicate experiments 
486 in different starting clones. For each clone, 40 replicate experiments were performed. Number of

487 mutants as determined by $\mathrm{CFU} / \mathrm{mL}$ were converted to an estimated mutation rate and a

488 corresponding 95\% confidence interval by the function "newton.LD” function in the R package

489 “rSalvador”(Zheng 2017).

491 DNA isolation and high-throughput sequencing. We conducted high resolution population

492 tracking by collecting $1 \mathrm{~mL}$ of culture at day $90,200,300,400,500,600,700,800$, and 900 of

493 experimental evolution. We used the DNeasy UltraClean Microbial Kit (Qiagen 12224; formerly

494 MO BIO UltraClean Microbial DNA Kit) to extract DNA. For library preparation and

495 sequencing, we submitted DNA to either the Hubbard Center for Genomic Analysis at the

496 University of New Hampshire, the Center for Genomics and Bioinformatics at Indiana

497 University, or the CLAS Genomics Facility at Arizona State University for library preparation

498 and sequencing. Library preparation was done by the Nextera DNA Library Preparation Kit

499 (Illumina, FC-121-1030) following an augmented protocol for optimization of reagent use

500 (Baym et al. 2015) before being pooled and sequenced as paired-end reads on an Illumina HiSeq

$5012500(\mathrm{UNH})$ or an Illumina NextSeq 500 (Indiana; ASU). The target depth is 100X.

503 Sequencing analysis. We performed Sequencing analysis on the Mason and Carbonate high-

504 performance computing clusters at Indiana University. The quality control of sequencing reads

505 were performed by Cutadapt v.1.9.1 (Martin 2011), which removes residual adapters and trims

506 low quality sequences. The qualified sequencing reads were then mapped to the Escherichia coli

507 K-12 substr. MG1655 reference genome (NC_000913.3). All mutations and their derived allele

508 frequencies (DAFs) were identified using Breseq v.0.30.2 with the predict-polymorphisms 
parameter setting (Deatherage and Barrick 2014). Furthermore, several criteria of further quality

510 checks were applied to the samples which we will only include in the following analysis: (1)

511 mean sequencing depths $>10$; (2) any WT sample identified to contain the 1,830 bp deletion in

512 mutL from the PMF5 progenitor strain was discarded; (3) regions lacking sequencing coverage

513 (i.e. depth $=0$ ) must be smaller than 5\% of the genome; and (4) the sequencing result should

514 reflect the correct genetic background in terms of ara markers, including a nonsynonymous SNP

515 at position 66528 , an intergenic SNP at position 70289 , and a multiple base substitution mutation

516 (SUB) at position 66533. For an ara + population, we required either of two SNPs showing DAF

$517<0.2$. For an ara-population, we required either of two SNPs showing DAF $>0.8$ or the SUB is

518 detected.

519 In the end, 396 genomic profiles passed QC and were included in the following analysis

520 (Table S3). In the case of M transfer size under WT background, only six out of eight replicates

521 of evolved populations are left for the following analysis because the other two were potentially

522 contaminated by MMR- strains. In the other five combinations of transfer size and genetic

523 background, there are still eight replicates of evolved populations for the following analysis. For

524 every evolved population subject to the following analysis, its sequencing profiles are available

525 in at least seven different time points.

526 To make sure that we do not use the mutations that originated from the starting clone

527 before experimental evolution in the analysis, we discarded any mutations with a DAF $=1.0$ at

528 one time point for at least 11 experimental populations with the same genetic background from

529 the analysis. Furthermore, the highly repetitive sequences in $r s x$ genes are known to cause errors

530 in SNP calling (McCloskey et al. 2018), so they were also discarded from the analysis. 
532 Number of guaranteed generations. Given the observation that carrying capacity of

533 experimental populations can be recovered within a transfer period (i.e. one day), the

534 guaranteed generation numbers in 900 days for dilution factors $=10^{-1}, 10^{-4}$, and $10^{-7}$ were

535 respectively estimated as 900 times of $\log _{2}(10), \log _{2}\left(10^{4}\right)$, and $\log _{2}\left(10^{7}\right)$, which is equal to

$536 \quad 3.0 \mathrm{k}, 12 \mathrm{k}$, and $21 \mathrm{k}$.

Rate of genomic evolution. The level of genomic divergence for each experimental population at each time point is defined by summing all DAFs of detected mutations. We then calculated the mean genomic divergence across all eligible experimental populations in each combination of

541 transfer size and genetic background. We further performed the linear regression by the function

542 "lm" in R with formula "mean genomic divergence $\sim$ guaranteed generations +0 ", which

543 enforces the $y$-intercept as 0 . The slope of the regression is the estimated rate of genomic

544 evolution. We also performed a nonlinear regression using the formula "mean genomic

545 divergence $\sim$ guaranteed generations + square root of guaranteed generations +0 ”, which was

546 previously proposed to catch the trend of diminishing returns (Tenaillon et al. 2016).

548 Identification of fixed mutations by hidden Markov chain. For each population, clade-aware

549 hidden Markov model (caHMM) was performed using a modified version (Behringer et al. 2020)

550 of previously released code (Good et al. 2017). For the populations in which caHMM can not

551 finish, we instead performed well-mixed hidden Markov chain (wmHMM) using a modified

552 version (Behringer et al. 2020) of previously released code (Good et al. 2017). The single clade

553 in wmHMM is defined as the basal clade. Fixed mutations are then defined as mutations that are

554 inferred to be fixed in basal, major, or minor clade in the results of either analysis. 
556 Estimation of selection coefficients. For each fixed mutation, we estimate its selection

557 coefficients by its temporal data of DAFs. If a mutation is in the basal clade, no correction is

558 needed. If caHMM infers a mutation belongs to the major or minor clade, its corrected allele

559 frequency will be the DAF devided by the proportion of the population belonging to the major or

560 minor clade (also inferred by caHMM). For each of two available consecutive time points $i$ and $j$,

561 if the corrected allele frequencies at both time points $\left(p_{\mathrm{i}}\right.$ and $\left.p_{\mathrm{j}}\right)$ are smaller than 0.95 and larger

562 than 0.05 , the selection coefficient is calculated as

$$
\ln \frac{p_{j}\left(1-p_{i}\right)}{p_{i}\left(1-p_{j}\right)} /\left(t_{j}-t_{i}\right)
$$

564 where $t_{\mathrm{i}}$ and $t_{\mathrm{j}}$ is the number of guaranteed generations at time point $i$ and $j$, respectively. The

565 negative values are discarded. The largest positive value across all pairs of time point are used as

566 the final measurement.

568 Calculation of neutrality index. As discussed in the main text, we defined $\left(F_{\mathrm{N}} / F_{\mathrm{S}}\right) /\left(U_{\mathrm{N}} / U_{\mathrm{S}}\right)$ as

569 the neutrality index of nonsynonymous SNPs, where $F_{\mathrm{N}}$ is the number of nonsynonymous SNPs

570 fixed within a clade or fixed in an entire population, $F_{\mathrm{S}}$ is the number of synonymous SNPs fixed

571 within a clade or fixed in an entire population, $U_{\mathrm{N}}$ is the number of nonsynonymous SNPs in the

572 mutation- accumulation experiment, and $U_{\mathrm{S}}$ is the number of synonymous SNPs in the mutation

573 accumulation-experiment. We also similarly defined $\left(F_{\mathrm{I}} / F_{\mathrm{S}}\right) /\left(U_{\mathrm{I}} / U_{\mathrm{S}}\right)$ as neutrality index of

574 intergenic SNPs, where $F_{\mathrm{I}}$ is the number of intergenic SNPs fixed within a clade or fixed in an

575 entire population, and $U_{\mathrm{I}}$ is the number of intergenic SNPs in the mutation-accumulation

576 experiment. The values of $U_{\mathrm{N}}, U_{\mathrm{S}}$, and $U_{\mathrm{I}}$ are from a previously published mutation-

577 accumulation experiment of our ancestral lines (Lee et al. 2012). We calculated population- 
578 specific indexes and then acquired population-wise mean and SE. The populations with $F_{\mathrm{N}}=0$

579 and $F_{\mathrm{I}}=0$ are discarded in the calculation of neutrality index of nonsynonymous and intergenic

580 SNPs, respectively.

581

582 Calculation of $\boldsymbol{G}$-scores. For each combination of genetic background and transfer size, we

583 quantified the parallelism of the fixed nonsynonymous mutations using the sum of $G$-scores

584 across genes (Tenaillon et al. 2016). A larger $G$-score for a gene suggests that the fixed

585 nonsynonymous mutations are more overrepresented in that gene. Specifically, to calculate a

586 genic $G$-score $\left(G_{\mathrm{i}}\right)$, we first counted the observed number of fixed nonsynonymous mutations in

587 gene $i\left(O_{\mathrm{i}}\right)$ per a combination of genetic background and transfer size. We then calculated the

588 expected number for gene $i\left(E_{\mathrm{i}}\right)$ by $O_{\mathrm{tot}}\left(L_{\mathrm{i}} / L_{\mathrm{tot}}\right)$, where $O_{\mathrm{tot}}=\Sigma_{\mathrm{i}} O_{\mathrm{i}}, L_{\mathrm{i}}$ is the number of

589 nonsynonymous sites for gene $i$, and $L_{\mathrm{tot}}=\Sigma_{\mathrm{i}} L_{\mathrm{i}}$. In the end, $G_{\mathrm{i}}$ is defined by $2 O_{\mathrm{i}} \ln \left(O_{\mathrm{i}} / E_{\mathrm{i}}\right)$ or

590 defined as zero when $O_{\mathrm{i}}=0$ or when $2 O_{\mathrm{i}} \ln \left(O_{\mathrm{i}} / E_{\mathrm{i}}\right)<0$.

It was noted that the null expectation of $G$-scores varies with total number of fixed

592 nonsynonymous mutations (Behringer et al. 2020). Therefore, for each combination of genetic

593 background and transfer size, we performed 20,000 simulations in each of which $O_{\text {tot }}$ hits are

594 randomly distributed among all $L_{\text {tot }}$ sites across all genes in the reference genome. Then the

595 significance of the sum of $G$-scores was evaluated by the $z$ score defined by (the observed sum -

596 mean of simulated sums) / (standard deviation of simulated sums).

598 Calculation of mean Bray-Curtis similarity. For each combination of genetic background and

599 transfer size, we also quantified the parallelism of the fixed nonsynonymous mutations using the

600 mean Bray-Curtis similarity across all pairs of experimental populations for a TP/GB 
combination (Turner et al. 2018; Behringer et al. 2020). Specifically, for a pair of populations $j$ and $k$, their Bray-Curtis similarity is defined by

$$
1-\frac{\sum_{i}\left|o_{i j}-o_{i k}\right|}{\sum_{i}\left(o_{i j}+o_{i k}\right)}
$$

604 where $o_{\mathrm{ij}}$ and $o_{\mathrm{ik}}$ is the observed number of fixed nonsynonymous mutations in gene $i$ for

605 population $j$ and $k$, respectively.

607 simulations to acquire the null distribution. In each simulation, we randomly sample the

608 nonsynonymous sites up to the number of observed fixed nonsynonymous mutations for each

609 population and calculated mean Bray-Curtis similarity as described above. After acquiring the

610 null distribution, we evaluated the significance of the observed mean Bray-Curtis similarity by

611 calculating the $z$ score defined by (the observed value - mean of simulated values) / (standard

612 deviation of simulated values).

614 Overrepresentation of the genes affected by nonsynonymous mutations. To evaluate the

615 significance of $G$-score for gene $i$, we directly compared the $G_{\mathrm{i}}$ to the distribution of 20,000

616 simulated $G_{\mathrm{i}}$, and the $P$-value was defined as the proportion of simulated $G_{\mathrm{i}}$ larger or equal to the

617 observed $G_{\text {i. }}$. For multiple test correction, we multiplied each gene's $P$-value by the number of

618 genes with at least one hit by the set of fixed nonsynonymous mutations (Bonferroni correction).

619 The genes are called significant only if the genes show Bonferroni corrected $P$-value $<0.05$.

621 Enrichment test of GO terms and KEGG pathways. Using the set of significant genes, we

622 performed the enrichment test of gene ontology terms using the function “enrichGO” in R 
623 package "DOSE" (Yu et al. 2015) with $q$-value cut-off $=0.05$ and the organismal database as

624 org.EcK12.eg.db. We also performed the enrichment test of KEGG pathways using the function

625 "enrichKEGG" in the same package but found no terms with $q$-value $<0.05$.

627 Overrepresentation of the intergenic regions affected by fixed mutations. The identification

628 of the intergenic regions affected by mutations was also performed by the way similar to identify

629 the genes affected by nonsynonymous fixed mutations in genic $G$-score approach (Tenaillon et

630 al. 2016). Instead of focusing on genic regions, genome-wide intergenic regions are focused. For

631 each combination of genetic background and transfer size, we first counted the observed number

632 of intergenic mutations in intergenic region $i\left(O_{\mathrm{i}}\right)$, and the expected number for intergenic region

$633 i\left(E_{\mathrm{i}}\right)$ was calculated by $O_{\mathrm{tot}}\left(L_{\mathrm{i}} / L_{\mathrm{tot}}\right)$, where $O_{\mathrm{tot}}=\Sigma_{\mathrm{i}} O_{\mathrm{i}}, L_{\mathrm{i}}$ is the length for intergenic region $i$, and

$634 L_{\mathrm{tot}}=\Sigma_{\mathrm{i}} L_{\mathrm{i}}$. The $G$-score for intergenic region $i\left(G_{\mathrm{i}}\right)$ was then calculated by $2 O_{\mathrm{i}} \ln \left(O_{\mathrm{i}} / E_{\mathrm{i}}\right)$,

635 following the methods described in the above section. We also performed 20,000 simulations

636 and determined the Bonferroni corrected $P$-value for each gene $i$ following the methods

637 described in the above section.

639 Overrepresentation of the genes affected by structural fixed mutations. The identification of

640 the genes affected by structural fixed mutations was performed by the way similar to identify the

641 genes affected by nonsynonymous fixed mutations in genic $G$-score approach (Tenaillon et al.

642 2016). Structural mutations include indels and IS-element insertions. For each combination of

643 genetic background and transfer size, we first counted the observed number of populations with

644 any structural mutations in gene $i\left(O_{\mathrm{i}}\right)$, and the expected number for gene $i\left(E_{\mathrm{i}}\right)$ was calculated by

$645 O_{\mathrm{tot}}\left(L_{\mathrm{i}} / L_{\mathrm{tot}}\right)$, where $O_{\mathrm{tot}}=\Sigma_{\mathrm{i}} O_{\mathrm{i}}, L_{\mathrm{i}}$ is the gene length for gene $i$, and $L_{\mathrm{tot}}=\Sigma_{\mathrm{i}} L_{\mathrm{i}}$. The $G$-score for 
gene $i\left(G_{\mathrm{i}}\right)$ was then calculated by $2 O_{\mathrm{i}} \ln \left(O_{\mathrm{i}} / E_{\mathrm{i}}\right)$, following the methods described in the above section.

We also performed 20,000 simulations and determined the Bonferroni corrected $P$-value

649 for each gene $i$ following the methods described in the above section. As a result, we found all

650 the genes with $O_{\mathrm{i}} \geq 2$ show Bonferroni corrected $P$-value $<0.05$.

652 Correlations between pairs of SNPs. For each evolved population, we focused on the

653 nonsynonymous, synonymous, and intergenic SNPs in which at least two nonzero DAFs were

654 found. For each pair of two such SNPs, we calculated the change of DAFs. Then we calculated

655 Pearson's correlation coefficients across only all the odd-numbered changes of DAFs to avoid

656 non-independence (Lynch and Ho 2020). That is to say, if a population has sequencing profiles

657 available for analysis at every sampling time points (days 90, 200, 300, 400, 500, 600, 700, 800,

658 900), we will calculate Pearson's correlation coefficients using the five changes of DAFs: the

659 one between day 0 and day 90, between day 200 and day 300, between day 400 and day 500,

660 between day 600 and day 700, and between day 800 and day 900 . For another example of

661 population, if its sequencing profile at day 90 is discard from analysis due to low quality, the

662 Pearson's correlation coefficients using only the four changes of DAFs: the one between day 0

663 and day 200, between day 300 and day 400, between day 500 and day 600, between day 700 and

664 day 800. Note that at least four changes of DAFs are used for each evolved population because

665 no populations have more than two missing profiles. We then get the distribution of Pearson's

666 correlation coefficients for each evolved population.

To establish the baseline for comparison in each combination of transfer size and genetic 
669 mutations from two different random populations with all sequencing profiles available (i.e. they

670 are unlinked for sure). We followed the same procedure above to calculate Pearson's correlation

671 coefficients for each pair of unlinked mutations. When simulating a distribution of Pearson's

672 correlation coefficients, we used 100 pair of unlinked mutations. We then repetitively performed

673100 rounds of simulation to get the mean and SE for the distribution.

674

675 Data Availability

676 Sequencing data generated during this study are available at NCBI's Sequencing Read Archive:

677 BioProject PRJNA532905 and PRJNA722381.

678

679 Code Availability

680 Codes generated to analyze sequencing data are available at

681 https://github.com/LynchLab/ECEE_Hypermutator

682

683 Acknowledgements

684 We thank D. Allan Drummond, Jay T. Lennon, and James B. McKinlay for their helpful

685 comments. High Performance Computing Resources were provided and maintained by the

686 National Center for Genome Analysis Support at Indiana University. This work was supported

687 by Army Research Office Grants ARO65308-LS-MU and W911NF-14-1-0411 (M. L.) and

688 National Institutes of Health Grant F32GM123703 (M. G. B.) and R35GM122566 (M. L.).

689

690 Author Contributions 
691 Conceptualization, W-C.H., M.G.B., and M.L.; Methodology, W-C.H., M.G.B., and S.F.M.;

692 Investigation, W-C.H., M.G.B., S.F.M., J.G., A.N., M.A., and G.F.B.; Formal Analysis, W-C.H.,

693 and M.G.B; Writing - Original Draft, W-C.H, and M.G.B.; Writing - Review \& Editing, W-

694 C.H., M.G.B., W-C.H., and M.L.; Visualization, W-C.H. and M.G.B.; Funding Acquisition,

695 M.G.B. and M.L.

696

697 Declaration of Interests

698 The authors declare no competing interests. 


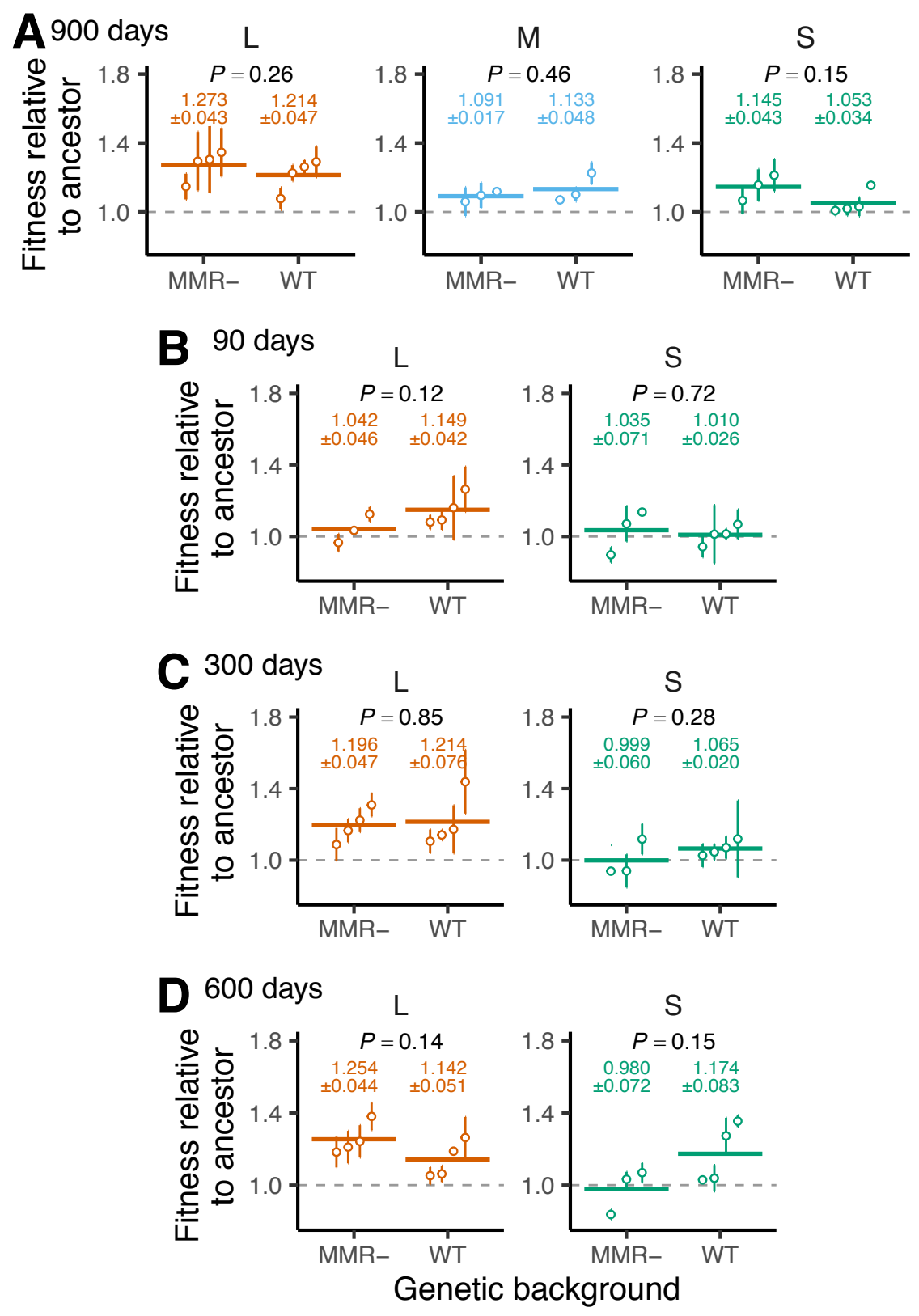

Figure 1. Fitness improvement during experimental evolution. For evolved populations under different transfer sizes (orange for L, blue for $\mathrm{M}$, or green for S) and different genetic backgrounds (MMR- or WT), mean fitnesses relative to the ancestor at (A) day 900, (B) day 90, (C) day 300, or (D) day 600 are reported. Each open circle represents an estimated mean for an evolved population with at least three independent competition assays. The error bars represent SEs. Gray dashed lines represent no improvement from ancestral fitness. The means across all evolved lines for a combination of transfer size and genetic background are represented by colored horizontal lines; the numeric values of means and SEs are printed on the top. The $P$-values for nested ANOVA are also shown on the top. 


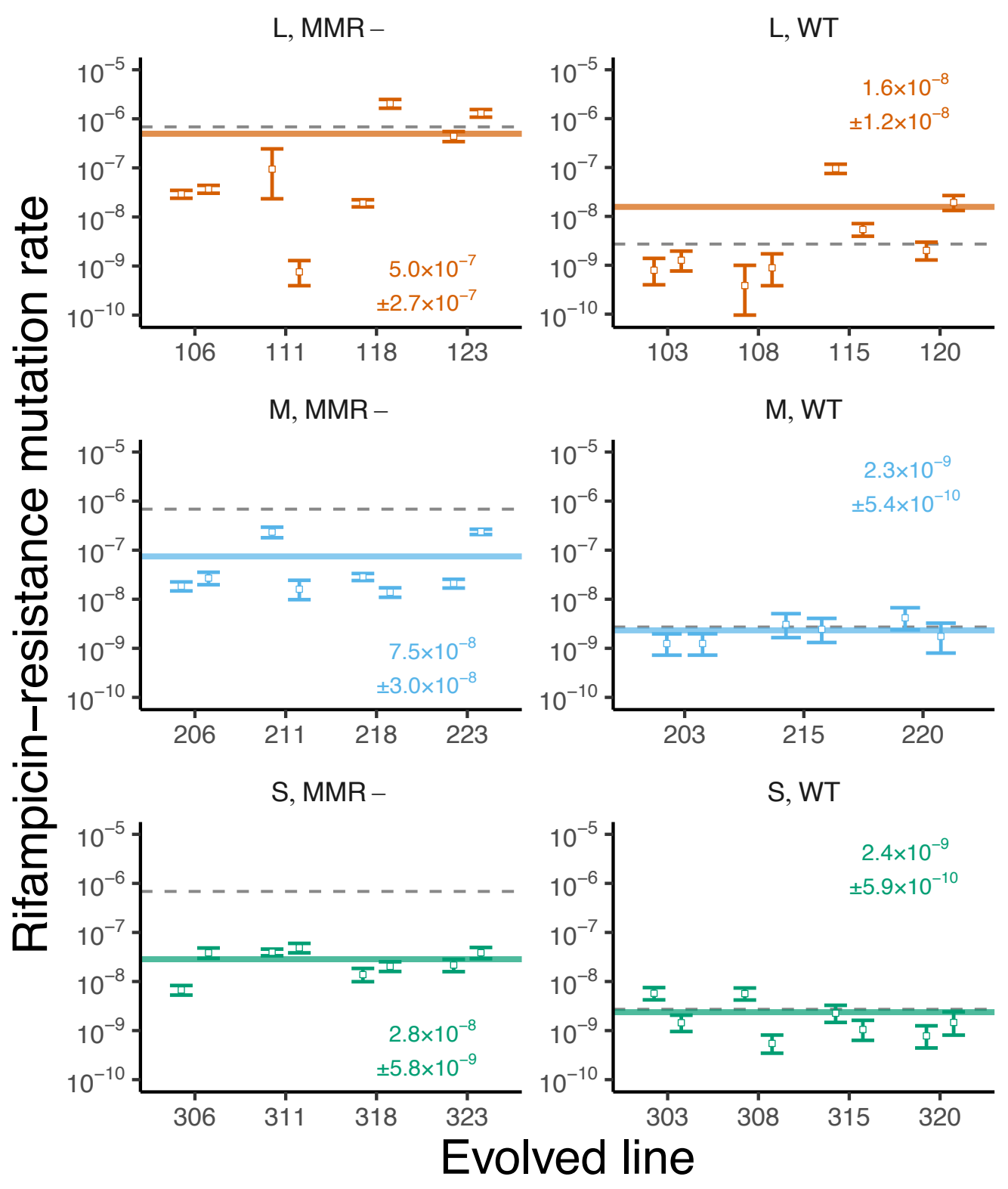

Figure 2. Evolution of mutation rates after 900 days of experimental evolution. Each panel shows mutation rates of evolved populations in different combinations of transfer sizes (L, M, or S) and genetic background (MMR- for mismatch repair defective or WT for wild-type). In each combination, three or four evolved populations were tested. Two clones per evolved population were isolated and measured. The open circles and error bars represent the mean and the 95\% confidence interval for each clone. The grey dashed line represents the mutation-rate measurement of the corresponding ancestor. The colored horizontal lines represent the mean mutation-rate measurement of each combination; the value of means and their SEs are also printed. 


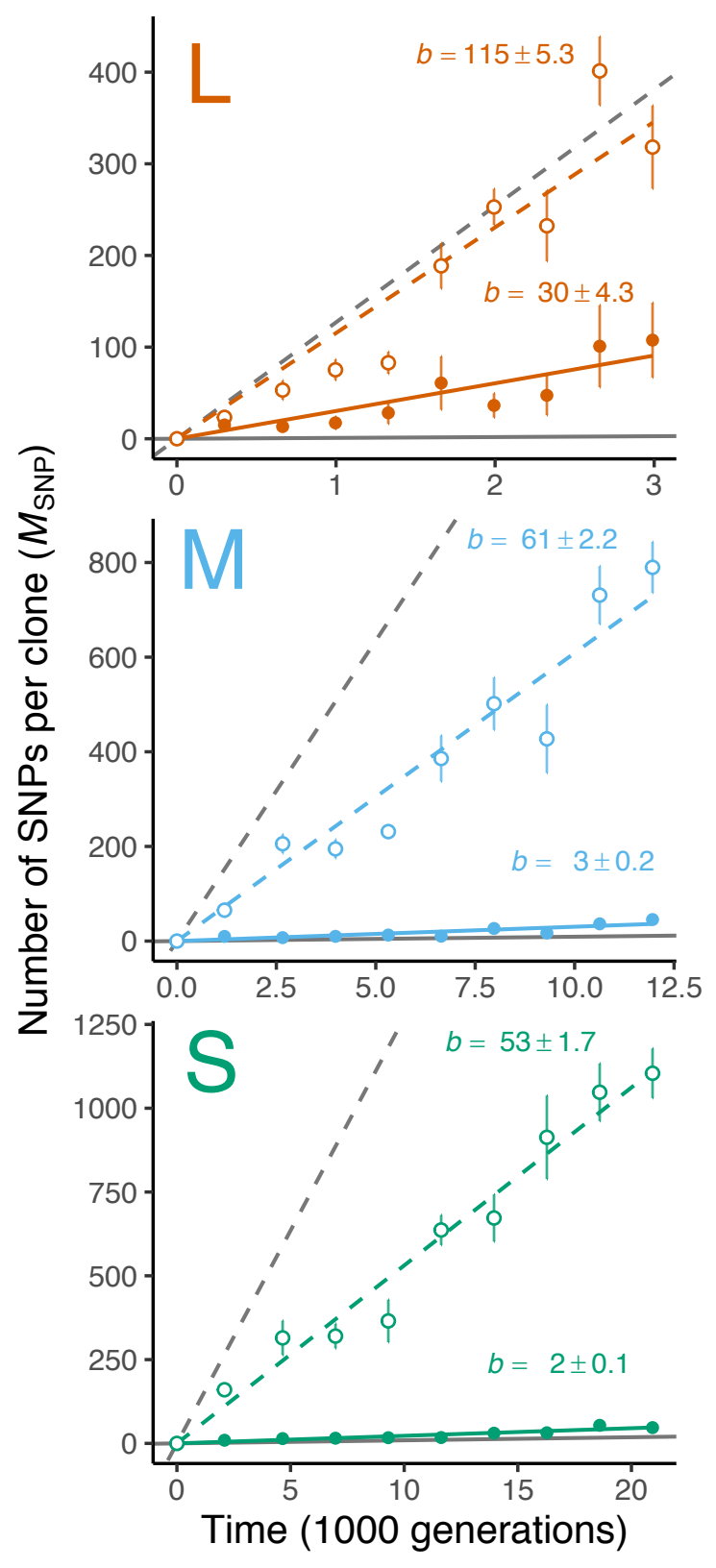

Figure 3. Rate of genomic evolution in evolved populations. Each panel shows the results of evolved populations in two genetic backgrounds (MMR- and WT) in a transfer size (L, M, or S). Each dot shows a mean number of SNPs per clone for MMR- (open circles) or WT (closed circles) populations at a sequencing time point. The error bars represent the associated standard errors. The dashed and solid lines are linear regressions against the time for MMR- and WT populations, respectively. The estimated slope $(b)$ and associated standard error are also printed for each regression line. The grey dashed and solid lines represent how evolved populations are expected to accumulate mutations based on the initial mutation rates of MMR- and WT ancestors. 

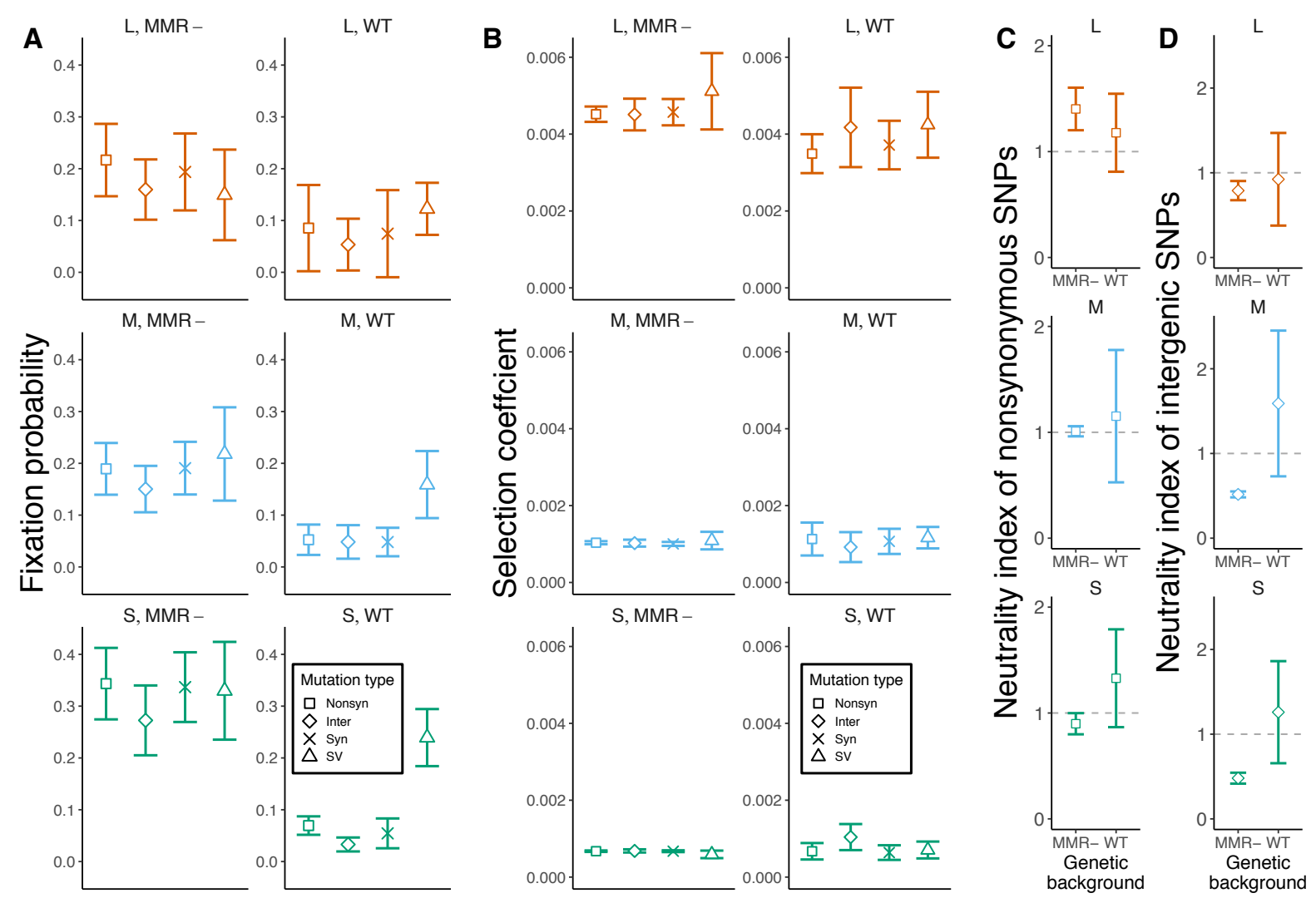

Figure 4. Analysis of strength of natural selection associated with fixed mutations in different categories in different treatments of experimental evolution. (A) Each symbol shows the population mean probability of nonsynonymous mutations (squares), intergenic mutations (diamonds), synonymous mutations (crosses), or structure variation mutations (SV; triangles) that reached within-clade fixation in each combination of transfer size $(\mathrm{L}, \mathrm{M}$, or $\mathrm{S})$ and genetic background (MMR- or WT). The error bars show the 95\% confidence intervals. (B) Each symbol shows the mean selection coefficient of nonsynonymous mutations (squares), intergenic mutations (diamonds), synonymous mutations (crosses), or SV (triangles) that are fixed in any clade in any population belonging in a combination of transfer size and genetic background. The error bars show the 95\% confidence intervals. (C) Each square shows the population mean neutrality index of nonsynonymous mutations for a combination of transfer size and genetic background. The error bars show the $95 \%$ confidence intervals. The grey lines indicate where the value $=1.0$. (D) Each square shows the population mean neutrality index of intergenic mutations for a combination of transfer size and genetic background. The error bars show the 95\% confidence intervals. The horizontal grey lines denote the point of neutrality (1.0). 

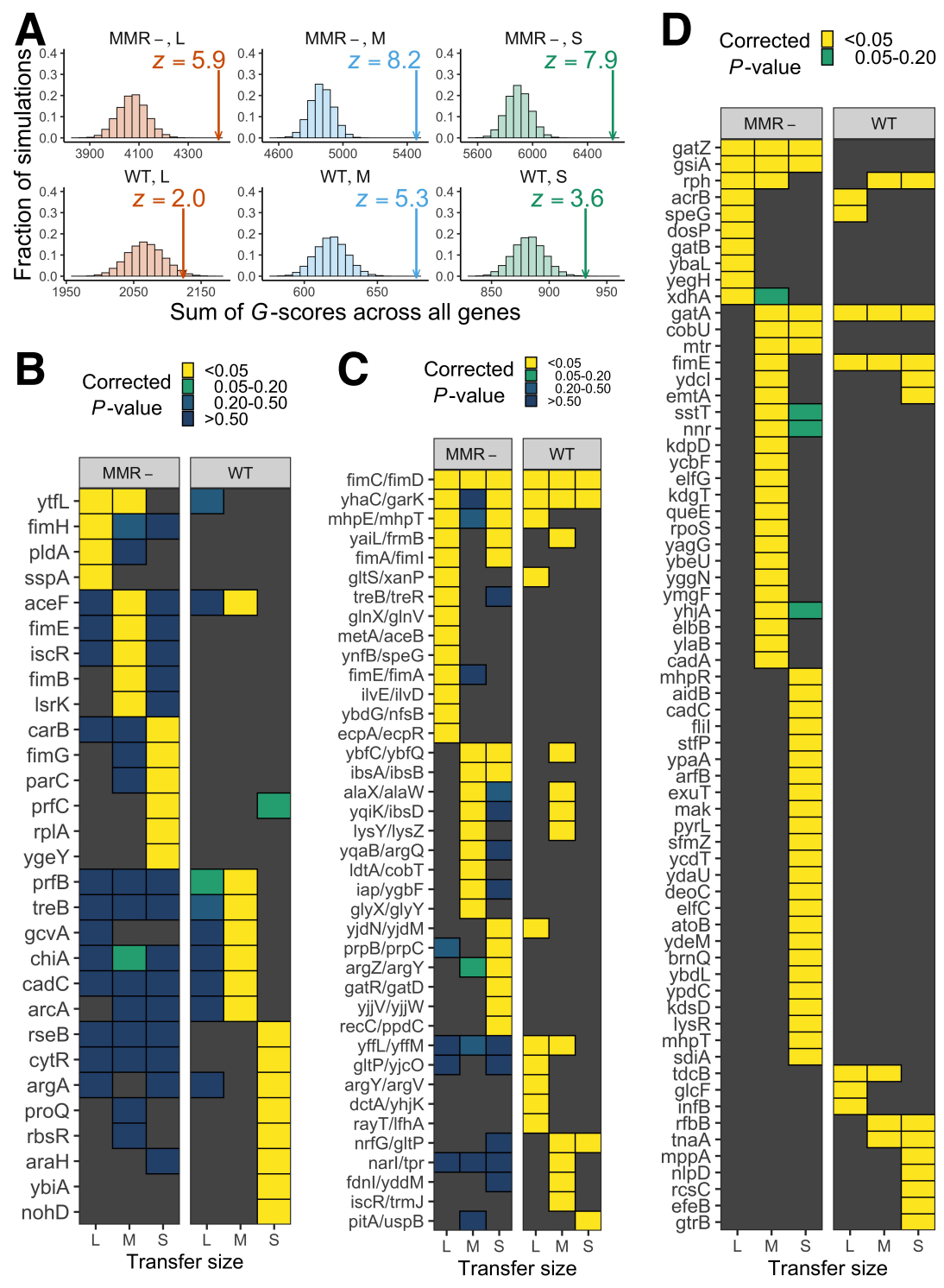

Figure 5. Evolutionary parallelism as evidence for positive selection. (A) The arrow and vertical dashed line show the observed sum of $G$-scores, representing the extent of parallel mutation for a particular combination of transfer size and genetic background. The histogram shows the distribution of 20,000 simulated sums of $G$-scores, representing the null distribution of evolutionary parallelism. The significance of the observed sums can be evaluated by $z$-scores $(z$ $>1.65$ for one-tailed $P<0.05$ ). (B) List of genes with fixed nonsynonymous mutations. Significance levels (simulated $P$-values with Bonferroni correction) are shown by the different non-black colours of tiles. Genes with no such hits in a particular combination are shown by black tiles. (C) List of genes with fixed intergenic mutations. Significance levels (simulated $P$ values with Bonferroni correction) are shown by the different non-black colours of tiles. Intergenic regions with no such hits in a particular combination are shown by black tiles. (D) List of genes significantly overrepresented for structural mutations that are likely under positive selection. Yellow tiles highlight genes observed in at least two populations and yielding simulated $P$-values $<0.05$ after Bonferroni correction. 


\section{References}

Abraham JM, Freitag CS, Clements JR, Eisenstein BI. 1985. An invertible element of DNA controls phase variation of type 1 fimbriae of Escherichia coli. Proc Natl Acad Sci US A 82: 5724-5727.

Arjan JA, Visser M, Zeyl CW, Gerrish PJ, Blanchard JL, Lenski RE. 1999. Diminishing returns from mutation supply rate in asexual populations. Science 283: 404-406.

Avalos Vizcarra I, Hosseini V, Kollmannsberger P, Meier S, Weber SS, Arnoldini M, Ackermann M, Vogel V. 2016. How type 1 fimbriae help Escherichia coli to evade extracellular antibiotics. Sci Rep 6: 18109.

Barrick JE, Yu DS, Yoon SH, Jeong H, Oh TK, Schneider D, Lenski RE, Kim JF. 2009. Genome evolution and adaptation in a long-term experiment with Escherichia coli. Nature 461: 12431247.

Barroso-Batista J, Sousa A, Lourenco M, Bergman ML, Sobral D, Demengeot J, Xavier KB, Gordo I. 2014. The first steps of adaptation of Escherichia coli to the gut are dominated by soft sweeps. PLoS Genet 10: e1004182.

Baym M, Kryazhimskiy S, Lieberman TD, Chung H, Desai MM, Kishony R. 2015. Inexpensive multiplexed library preparation for megabase-sized genomes. PLoS One 10: e0128036. Behringer MG, Choi BI, Miller SF, Doak TG, Karty JA, Guo W, Lynch M. 2018. Escherichia coli cultures maintain stable subpopulation structure during long-term evolution. Proc Natl Acad Sci U S A 115: E4642-E4650.

Behringer MG, Ho W-C, Miller SF, Meraz JC, Boyer GF, Lynch M. 2020. Evolutionary response to resource deprivation: parallelism and nonmonotonicity. bioRxiv: 865584 . 
Bell AW, Buckel SD, Groarke JM, Hope JN, Kingsley DH, Hermodson MA. 1986. The nucleotide sequences of the rbsD, rbsA, and rbsC genes of Escherichia coli K12. J Biol Chem 261: 7652-7658.

Bollback JP, Huelsenbeck JP. 2007. Clonal interference is alleviated by high mutation rates in large populations. Mol Biol Evol 24: 1397-1406.

Campos PR, Wahl LM. 2010. The adaptation rate of asexuals: deleterious mutations, clonal interference and population bottlenecks. Evolution 64: 1973-1983.

Couce A, Alonso-Rodriguez N, Costas C, Oliver A, Blazquez J. 2016. Intrapopulation variability in mutator prevalence among urinary tract infection isolates of Escherichia coli. Clin Microbiol Infect 22: 566 e561-567.

Couce A, Caudwell LV, Feinauer C, Hindre T, Feugeas JP, Weigt M, Lenski RE, Schneider D, Tenaillon O. 2017. Mutator genomes decay, despite sustained fitness gains, in a long-term experiment with bacteria. Proc Natl Acad Sci U S A 114: E9026-E9035.

Crow JF, Kimura M. 1965. Evolution in sexual and asexual populations. The American Naturalist 99: 439-450.

Deatherage DE, Barrick JE. 2014. Identification of mutations in laboratory-evolved microbes from next-generation sequencing data using breseq. Methods Mol Biol 1151: 165-188.

Desai MM, Fisher DS. 2007. Beneficial mutation selection balance and the effect of linkage on positive selection. Genetics 176: 1759-1798.

Desai MM, Fisher DS, Murray AW. 2007. The speed of evolution and maintenance of variation in asexual populations. Curr Biol 17: 385-394.

Domka J, Lee J, Bansal T, Wood TK. 2007. Temporal gene-expression in Escherichia coli K-12 biofilms. Environ Microbiol 9: 332-346. 
Foster PL. 2006. Methods for determining spontaneous mutation rates. Methods Enzymol 409:

195-213.

Gerrish PJ, Lenski RE. 1998. The fate of competing beneficial mutations in an asexual population. Genetica 102-103: 127-144.

Gillespie JH. 2000. Genetic drift in an infinite population. The pseudohitchhiking model. Genetics 155: 909-919.

Giraud A, Matic I, Tenaillon O, Clara A, Radman M, Fons M, Taddei F. 2001. Costs and benefits of high mutation rates: adaptive evolution of bacteria in the mouse gut. Science 291: 2606-2608.

Good BH, Rouzine IM, Balick DJ, Hallatschek O, Desai MM. 2012. Distribution of fixed beneficial mutations and the rate of adaptation in asexual populations. Proc Natl Acad Sci US A 109: 4950-4955.

Good BH, McDonald MJ, Barrick JE, Lenski RE, Desai MM. 2017. The dynamics of molecular evolution over 60,000 generations. Nature 551: 45-50.

Guo Y, Vucelja M, Amir A. 2019. Stochastic tunneling across fitness valleys can give rise to a logarithmic long-term fitness trajectory. Sci Adv 5: eaav3842.

Handel A, Rozen DE. 2009. The impact of population size on the evolution of asexual microbes on smooth versus rugged fitness landscapes. BMC Evol Biol 9: 236.

Iuchi S, Cameron DC, Lin EC. 1989. A second global regulator gene (arcB) mediating repression of enzymes in aerobic pathways of Escherichia coli. J Bacteriol 171: 868-873.

Kaper JB, Nataro JP, Mobley HL. 2004. Pathogenic Escherichia coli. Nat Rev Microbiol 2: 123140. 
Kim Y, Orr HA. 2005. Adaptation in sexuals vs. asexuals: clonal interference and the FisherMuller model. Genetics 171: 1377-1386.

Kimura M. 1967. On the evolutionary adjustment of spontaneous mutation rates. Genetical Research 9: 23-34.

Kosheleva K, Desai MM. 2013. The dynamics of genetic draft in rapidly adapting populations. Genetics 195: 1007-1025.

Kristensen HH, Valentin-Hansen P, Sogaard-Andersen L. 1996. CytR/cAMP-CRP nucleoprotein formation in E. coli: the CytR repressor binds its operator as a stable dimer in a ternary complex with cAMP-CRP. J Mol Biol 260: 113-119.

Lange R, Hengge-Aronis R. 1991. Identification of a central regulator of stationary-phase gene expression in Escherichia coli. Mol Microbiol 5: 49-59.

Le Trong I, Aprikian P, Kidd BA, Forero-Shelton M, Tchesnokova V, Rajagopal P, Rodriguez V, Interlandi G, Klevit R, Vogel V, Stenkamp RE, Sokurenko EV, Thomas WE. 2010. Structural basis for mechanical force regulation of the adhesin FimH via finger trap-like beta sheet twisting. Cell 141: 645-655.

Lee H, Popodi E, Tang H, Foster PL. 2012. Rate and molecular spectrum of spontaneous mutations in the bacterium Escherichia coli as determined by whole-genome sequencing. Proc Natl Acad Sci U S A 109: E2774-2783.

Lenski RE, Rose MR, Simpson SC, Tadler SC. 1991. Long-Term experimental evolution in Escherichia coli. I. adaptation and divergence during 2,000 generations. The American

Naturalist 138: 1315-1341. 
Li J, Attila C, Wang L, Wood TK, Valdes JJ, Bentley WE. 2007. Quorum sensing in Escherichia coli is signaled by AI-2/LsrR: effects on small RNA and biofilm architecture. J Bacteriol 189: 6011-6020.

Long H, Miller SF, Strauss C, Zhao C, Cheng L, Ye Z, Griffin K, Te R, Lee H, Chen CC, Lynch M. 2016. Antibiotic treatment enhances the genome-wide mutation rate of target cells. Proc Natl Acad Sci U S A 113: E2498-E2505.

Lynch M. 2008. The cellular, developmental and population-genetic determinants of mutationrate evolution. Genetics 180: 933-943.

Lynch M. 2010a. Scaling expectations for the time to establishment of complex adaptations. Proc Natl Acad Sci U S A 107: 16577-16582.

Lynch M. 2010b. Evolution of the mutation rate. Trends Genet 26: 345-352.

Lynch M, Ackerman MS, Gout JF, Long H, Sung W, Thomas WK, Foster PL. 2016. Genetic drift, selection and the evolution of the mutation rate. Nat Rev Genet 17: 704-714.

Lynch M, Ho W-C. 2020. The limits to estimating population-genetic parameters with temporal data. Genome Biol Evol 12: 443-455.

Martin M. 2011. Cutadapt removes adapter sequences from high-throughput sequencing reads. EMBnet.journal 17: 10-12.

Mauzy CA, Hermodson MA. 1992. Structural homology between rbs repressor and ribose binding protein implies functional similarity. Protein Sci 1: 843-849.

McCloskey D, Xu S, Sandberg TE, Brunk E, Hefner Y, Szubin R, Feist AM, Palsson BO. 2018. Evolution of gene knockout strains of E. coli reveal regulatory architectures governed by metabolism. Nat Commun 9: 3796. 
McDonald JH, Kreitman M. 1991. Adaptive protein evolution at the Adh locus in Drosophila.

Nature 351: 652-654.

McDonald MJ, Hsieh YY, Yu YH, Chang SL, Leu JY. 2012. The evolution of low mutation rates in experimental mutator populations of Saccharomyces cerevisiae. Curr Biol 22: 12351240.

McDonald MJ. 2019. Microbial experimental evolution - a proving ground for evolutionary theory and a tool for discovery. EMBO Rep 20: e46992.

Mehta HH, Prater AG, Beabout K, Elworth RAL, Karavis M, Gibbons HS, Shamoo Y. 2019. The essential role of hypermutation in rapid adaptation to antibiotic stress. Antimicrob Agents Chemother 63.

Missiakas D, Mayer MP, Lemaire M, Georgopoulos C, Raina S. 1997. Modulation of the Escherichia coli sigmaE (RpoE) heat-shock transcription-factor activity by the RseA, RseB and RseC proteins. Mol Microbiol 24: 355-371.

Monod J. 1949. The growth of bacterial cultures. Annual Review of Microbiology 3: 371-394.

Muller HJ. 1932. Some genetic aspects of sex. The American Naturalist 66: 118-138.

Muller HJ. 1950. Our load of mutations. Am J Hum Genet 2: 111-176.

Navarro Llorens JM, Tormo A, Martinez-Garcia E. 2010. Stationary phase in gram-negative bacteria. FEMS Microbiol Rev 34: 476-495.

Naves P, del Prado G, Huelves L, Gracia M, Ruiz V, Blanco J, Dahbi G, Blanco M, Ponte Mdel C, Soriano F. 2008. Correlation between virulence factors and in vitro biofilm formation by Escherichia coli strains. Microb Pathog 45: 86-91.

Neher RA. 2013. Genetic draft, selective interference, and population genetics of rapid adaptation. Annu Rev Ecol Evol Syst 44: 195-215. 
Ochs IE, Desai MM. 2015. The competition between simple and complex evolutionary trajectories in asexual populations. BMC Evol Biol 15: 55.

Olsen PB, Schembri MA, Gally DL, Klemm P. 1998. Differential temperature modulation by HNS of the fimB and fimE recombinase genes which control the orientation of the type 1 fimbrial phase switch. FEMS Microbiol Lett 162: 17-23.

Park SC, Krug J. 2007. Clonal interference in large populations. Proc Natl Acad Sci U S A 104: $18135-18140$.

Park SC, Simon D, Krug J. 2010. The speed of evolution in large asexual populations. Journal of Statistical Physics 138: 381-410.

Penisson S, Singh T, Sniegowski P, Gerrish P. 2017. Dynamics and fate of beneficial mutations under lineage contamination by linked deleterious mutations. Genetics 205: 1305-1318.

Pletnev P, Osterman I, Sergiev P, Bogdanov A, Dontsova O. 2015. Survival guide: Escherichia coli in the stationary phase. Acta Naturae 7: 22-33.

Pratt LA, Kolter R. 1998. Genetic analysis of Escherichia coli biofilm formation: roles of flagella, motility, chemotaxis and type I pili. Mol Microbiol 30: 285-293.

Rand DM, Kann LM. 1996. Excess amino acid polymorphism in mitochondrial DNA: contrasts among genes from Drosophila, mice, and humans. Mol Biol Evol 13: 735-748.

Raynes Y, Halstead AL, Sniegowski PD. 2014. The effect of population bottlenecks on mutation rate evolution in asexual populations. J Evol Biol 27: 161-169.

Rolfe MD, Ter Beek A, Graham AI, Trotter EW, Asif HM, Sanguinetti G, de Mattos JT, Poole RK, Green J. 2011. Transcript profiling and inference of Escherichia coli K-12 ArcA activity across the range of physiologically relevant oxygen concentrations. J Biol Chem 286: $10147-$ 10154. 
Rossi E, Cimdins A, Luthje P, Brauner A, Sjoling A, Landini P, Romling U. 2018. "It's a gut feeling" - Escherichia coli biofilm formation in the gastrointestinal tract environment. Crit Rev Microbiol 44: 1-30.

Schembri MA, Kjaergaard K, Sokurenko EV, Klemm P. 2001. Molecular characterization of the Escherichia coli FimH adhesin. J Infect Dis 183 Suppl 1: S28-31.

Schiffels S, Szollosi GJ, Mustonen V, Lassig M. 2011. Emergent neutrality in adaptive asexual evolution. Genetics 189: 1361-1375.

Sezonov G, Joseleau-Petit D, D'Ari R. 2007. Escherichia coli physiology in Luria-Bertani broth. J Bacteriol 189: 8746-8749.

Sharma G, Sharma S, Sharma P, Chandola D, Dang S, Gupta S, Gabrani R. 2016. Escherichia coli biofilm: development and therapeutic strategies. J Appl Microbiol 121: 309-319.

Sheidy DT, Zielke RA. 2013. Analysis and expansion of the role of the Escherichia coli protein ProQ. PLoS One 8: e79656.

Shewaramani S, Finn TJ, Leahy SC, Kassen R, Rainey PB, Moon CD. 2017. Anaerobically grown Escherichia coli has an enhanced mutation rate and distinct mutational spectra. PLoS Genet 13: e1006570.

Sniegowski PD, Gerrish PJ, Lenski RE. 1997. Evolution of high mutation rates in experimental populations of E. coli. Nature 387: 703-705.

Spears PA, Schauer D, Orndorff PE. 1986. Metastable regulation of type 1 piliation in Escherichia coli and isolation and characterization of a phenotypically stable mutant. J Bacteriol 168: 179-185.

Sprouffske K, Aguilar-Rodriguez J, Sniegowski P, Wagner A. 2018. High mutation rates limit evolutionary adaptation in Escherichia coli. PLoS Genet 14: e1007324. 
Sung W, Ackerman MS, Miller SF, Doak TG, Lynch M. 2012. Drift-barrier hypothesis and mutation-rate evolution. Proc Natl Acad Sci U S A 109: 18488-18492.

Swings T, Van den Bergh B, Wuyts S, Oeyen E, Voordeckers K, Verstrepen KJ, Fauvart M, Verstraeten N, Michiels J. 2017. Adaptive tuning of mutation rates allows fast response to lethal stress in Escherichia coli. Elife 6.

Tachida H. 2000. DNA evolution under weak selection. Gene 261: 3-9.

Tarnita CE. 2017. The ecology and evolution of social behavior in microbes. J Exp Biol 220: 1824.

Tenaillon O, Toupance B, Le Nagard H, Taddei F, Godelle B. 1999. Mutators, population size, adaptive landscape and the adaptation of asexual populations of bacteria. Genetics 152: 485-493. Tenaillon O, Taddei F, Radmian M, Matic I. 2001. Second-order selection in bacterial evolution: selection acting on mutation and recombination rates in the course of adaptation. Res Microbiol 152: $11-16$.

Tenaillon O, Barrick JE, Ribeck N, Deatherage DE, Blanchard JL, Dasgupta A, Wu GC, Wielgoss S, Cruveiller S, Medigue C, Schneider D, Lenski RE. 2016. Tempo and mode of genome evolution in a 50,000-generation experiment. Nature 536: 165-170.

Turner CB, Marshall CW, Cooper VS. 2018. Parallel genetic adaptation across environments differing in mode of growth or resource availability. Evol Lett 2: 355-367.

Van den Bergh B, Swings T, Fauvart M, Michiels J. 2018. Experimental design, population dynamics, and diversity in microbial experimental evolution. Microbiol Mol Biol Rev 82. Veschetti L, Sandri A, Johansen HK, Lleo MM, Malerba G. 2020. Hypermutation as an evolutionary mechanism for Achromobacter xylosoxidans in cystic fibrosis lung Infection. Pathogens 9. 
Vogwill T, Phillips RL, Gifford DR, MacLean RC. 2016. Divergent evolution peaks under intermediate population bottlenecks during bacterial experimental evolution. Proc Biol Sci 283. Waksman G, Hultgren SJ. 2009. Structural biology of the chaperone-usher pathway of pilus biogenesis. Nat Rev Microbiol 7: 765-774.

Watson N, Dunyak DS, Rosey EL, Slonczewski JL, Olson ER. 1992. Identification of elements involved in transcriptional regulation of the Escherichia coli cad operon by external $\mathrm{pH} . J$ Bacteriol 174: 530-540.

Wein T, Dagan T. 2019. The effect of population bottleneck size and selective regime on genetic diversity and evolvability in bacteria. Genome Biol Evol 11: 3283-3290.

Wielgoss S, Barrick JE, Tenaillon O, Wiser MJ, Dittmar WJ, Cruveiller S, Chane-Woon-Ming B, Medigue C, Lenski RE, Schneider D. 2013. Mutation rate dynamics in a bacterial population reflect tension between adaptation and genetic load. Proc Natl Acad Sci US A 110: 222-227. Wilke CO. 2004. The speed of adaptation in large asexual populations. Genetics 167: 2045-2053. Williams AB, Foster PL. 2012. Stress-Induced Mutagenesis. EcoSal Plus 5.

Williams MD, Ouyang TX, Flickinger MC. 1994. Starvation-induced expression of SspA and SspB: the effects of a null mutation in sspA on Escherichia coli protein synthesis and survival during growth and prolonged starvation. Mol Microbiol 11: 1029-1043.

Yu GC, Wang LG, Yan GR, He QY. 2015. DOSE: an R/Bioconductor package for disease ontology semantic and enrichment analysis. Bioinformatics 31: 608-609.

Zheng Q. 2017. rSalvador: An R package for the fluctuation experiment. G3-Genes Genom Genet 7: 3849-3856. 